\title{
On possible generalization of the superstring action to eleven dimensions
}

\author{
A.A. Deriglazov* and A.V. Galajinsky ${ }^{\dagger}$ \\ Instituto de Física, Universidade de São Paulo, \\ P.O. Box 66318, 05315-970, São Paulo, SP, Brasil
}

\begin{abstract}
We suggest a $D=11$ super Poincaré invariant action for the superstring which has free dynamics in the physical variables sector. Instead of the standard approach based on the searching for an action with local $\kappa$-symmetry (or, equivalently, with corresponding first class constraints), we propose a theory with fermionic constraints of second class only. Then the $\kappa$-symmetry and the well known $\Gamma$-matrix identities are not necessary for the construction. Thus, at the classical level, the superstring action of the type described can exist in any spacetime dimensions and the known brane-scan can be revisited.
\end{abstract}

PAC codes: 0460D; 1130C; 1125

Keywords: eleven dimensional superstring, covariant quantization.

\section{Introduction}

A revival of interest to the problem of covariant formulation for eleven dimensional superstring is due to the search for M-theory (see Refs. 1-5 and references therein) which is expected to be the underlying quantum theory for the known extended objects. In the strong coupling limit of M-theory $R^{11} \rightarrow \infty$, where $R^{11}$ is the radius of 11th dimension, the vacuum is eleven dimensional Minkowski and the effective field theory is $D=11$ supergravity. Up to date, $D=11$ supergravity is viewed as the strong coupling limit of the ten dimensional type IIA superstring. Since $D=11$ super Poincaré symmetry survives in this special point in the moduli space of M-theory vacua ("uncompactified M-theory" according to Ref. 5), one may ask of the existence of a consistent D11 quantum theory

\footnotetext{
*deriglaz@fma.if.usp.br

†galajin@fma.if.usp.br
} 
with $D=11$ supergravity being its low energy limit. One possibility is the supermembrane action $\mathrm{\theta}$, but in this case one faces with the problem of a continuous spectrum for the first quantized supermembrand 9 . By analogy with the ten dimensional case, where the known supersymmetric field theories can be obtained as the low energy limit of the corresponding superstrings theory. But the problem is that a covariant formulation for $D=11$ superstring action is unknown even at the classical level. The classical Green-Schwarz (GS) superstring (with manifest space-time supersymmetry and local $\kappa$-symmetry) can propagate in 3,4 , 6 and 10 spacetime dimensions 11 and the standard approach fails to construct a $D=11$ superstring action.

The crucial ingredient in the construction of the GS superstring action is the $\Gamma$-matrix identity

$$
\Gamma_{\alpha(\beta}^{\mu}\left(C \Gamma^{\mu}\right)_{\gamma \delta)}=0
$$

It provides the existence of both global supersymmetry and local $\kappa$-symmetry for the action 1112. The $\kappa$-symmetry, in its turn, eliminates half of the initial $\theta$-variables as well as provides free dynamics in the physical variables sector. In this paper we discuss a possibility to construct a classical superstring action with those two properties in eleven dimensions. Subsequent development of our method may shed light on the problem of constructing the corresponding quantum theory. To elucidate the construction which will be suggested below let us discuss the problem in the Hamiltonian framework, where one finds the well known fermionic constraints $L_{\alpha}=0$ (see, for example, Refs. 11 and 12) which obey the Poisson brackets

$$
\left\{L_{\alpha}, L_{\beta}\right\}=2 i\left(\hat{p}^{\mu}+\Pi_{1}^{\mu}\right) \Gamma_{\alpha \beta}^{\mu} \delta\left(\sigma-\sigma^{\prime}\right)-2 \bar{\theta}^{\gamma} \partial_{1} \theta^{\delta} \Gamma_{\gamma(\delta}^{\mu}\left(C \Gamma^{\mu}\right)_{\alpha \beta)} \delta\left(\sigma-\sigma^{\prime}\right) .
$$

By virtue of Eq. (1), the last term in Eq. (2) vanishes for $D=3,4,6,10$. The resulting equation then means that half of the constraints are first class, which exactly corresponds to the $\kappa$-symmetry presented in the Lagrangian framework.

The next step is to impose an appropriate gauge. Then the following set of functions:

$$
\begin{aligned}
& L_{\alpha}=0, \\
& \Gamma^{+} \theta=0,
\end{aligned}
$$

is a system of second class (even through Eq. (1) has not been used). 
The situation changes drastically for the $D=11$ case, where instead of Eq. (1) one finds 13 国

$$
10 \Gamma_{\alpha(\beta}^{\mu}\left(C \Gamma^{\mu}\right)_{\gamma \delta)}+\Gamma_{\alpha(\beta}^{\mu \nu}\left(C \Gamma^{\mu \nu}\right)_{\gamma \delta)}=0 .
$$

Being appropriate for the construction of the supermembrane action [6], this identity does not allow one to formulate a $D=11$ superstring with desirable properties. As was shown by Curtright ${ }^{13}$, the globally supersymmetric action based on this identity involves additional to $x^{i}, \theta_{a}, \bar{\theta}_{\dot{a}}$ degrees of freedom in the physical sector. Moreover, it does not possess a $\kappa$-symmetry that could provide free dynamics 3 回 3 .

In this paper we suggest a $D=11$ super Poincaré invariant action for the classical superstring which has free dynamics in the physical variables sector. Instead of the standard approach based on the searching for an action with local $\kappa$-symmetry (or, equivalently, with corresponding first class constraints), we present a theory in which covariant constraints like Eqs. (3), (4) arise among others. Since it is a system of second class constraints, $\kappa$-symmetry and the identity (5) are not necessary for the construction. Thus, at the classical level, a superstring of the type described can exist in any spacetime dimension and the known brane scan can be revisited. For definiteness, in this paper we discuss the $D=11$ case only.

Two comments are in order. First, one needs to covariantize Eq. (4). The simplest possibility is to introduce an auxiliary variable $\Lambda^{\mu}(\tau, \sigma)$ subject to $\Lambda^{2}=0$ and replace Eq. (4) by $\Lambda_{\mu} \Gamma^{\mu} \theta=0$. The most preferable formulation seems to be that in which the gauge $\Lambda^{-}=1$ is possible. Then Eq. (4) is reproduced. Unfortunately, it seem to be impossible to introduce a pure gauge variable with the desired properties 16 20. Below, we present a formulation in which only zero modes of auxiliary variables survive in the sector of physical degrees of freedom. Since states spectrum of a string is determined by the action on the vacuum of oscillator modes only, one can expect that the presence of the zero modes will be inessential for the case. This fact will be demonstrated within the canonical quantization framework in Sec. 2 and 4.

Second, one expects that a model with constraints like Eqs. (3), (4) will possess (if any) off-shell super Poincaré symmetry in a nonstandard realization. Actually, global supersymmetry which does not spoil the equation $\Lambda_{\mu} \Gamma^{\mu} \theta=0$ looks like $\delta \theta \sim \Lambda_{\mu} \Gamma^{\mu} \epsilon$. On-shell, where $\Lambda^{2}=0$, only half of the supersymmetry parameters $\epsilon^{\alpha}$ are essential.

It is worth mentioning another motivation for this work. As was shown in Refs. 21-25, an action for the super $D$-brane allowing for the local $\kappa$-symmetry is very complicated. 
One can hope that our method being applied to that case, will lead to a more simple formulation.

The work is organized as follows. In Sec. 2 we present and discuss an action for the auxiliary variable $\Lambda^{\mu}$, which proves to be a necessary ingredient of our construction. In Sec. 3 a covariant action for the eleven dimensional superstring and its local symmetries are presented. In Sec. 4 within the framework of the Hamiltonian approach we prove that it has free dynamics. In Sec. 5 the role of the Wess-Zumino term presented in the action is elucidated. In Sec. 6 off-shell realization of the super Poincaré algebra is derived and discussed. Appendix contains our spinor convention for $D=11$.

\section{Action for auxiliary variables and their dynamics}

As was mentioned in the Introduction, we need to get in our disposal an auxiliary lightlike variable. So, as a preliminary step of our construction, let us discuss the following $D=11$ Poincaré invariant action

$$
S=-\int d^{2} \sigma\left[\Lambda^{\mu} \varepsilon^{a b} \partial_{a} A_{b}^{\mu}+\frac{1}{\phi} \Lambda^{\mu} \Lambda^{\mu}\right],
$$

which turns out to be a building block of the eleven dimensional superstring action considered below. Here $\Lambda^{\mu}\left(\sigma^{a}\right)$ is a $D=11$ vector and a $d 2$ scalar, $A_{a}^{\mu}\left(\sigma^{b}\right)$ is a $D=11$ and $d 2$ vector, while $\phi\left(\sigma^{a}\right)$ is a scalar field. In Eq. (6) we have set $\varepsilon^{a b}=-\varepsilon^{b a}, \varepsilon^{01}=-1$ and it was also supposed that $\sigma^{1} \subset[0, \pi]$. From the equation of motion $\delta S / \delta \phi=0$ it follows that $\Lambda^{\mu}$ is a light-like vector.

Local symmetries of the action are $d=2$ reparametrizations $\rrbracket$ and the following transformations with the parameters $\xi^{\mu}\left(\sigma^{a}\right), \omega_{a}\left(\sigma^{b}\right)$ :

$$
\begin{aligned}
& \delta_{\xi} A_{a}^{\mu}=\partial_{a} \xi^{\mu} \\
& \delta_{\omega} A_{a}^{\mu}=\omega_{a} \Lambda^{\mu} \\
& \delta_{\omega} \phi=\frac{1}{2} \phi^{2} \varepsilon^{a b} \partial_{a} \omega_{b} .
\end{aligned}
$$

\footnotetext{
${ }^{1}$ Note that the coupling to $d=2$ metric $g^{a b}\left(\sigma^{c}\right)$ is not necessary due to the presence of the $\varepsilon^{a b}$ symbol and the supposition that the variable $\phi$ transforms as a density $\phi^{\prime}\left(\sigma^{\prime}\right)=\operatorname{det}\left(\partial \sigma^{\prime} / \partial \sigma\right) \phi(\sigma)$ under reparametrizations.
} 
These symmetries are reducible because their combination with the parameters of a special form: $\omega_{a}=\partial_{a} \omega, \xi^{\mu}=-\omega \Lambda^{\mu}$ is a trivial symmetry: $\delta_{\omega} A_{a}^{\mu}=-\omega \partial_{a} \Lambda^{\mu}, \delta_{\omega} \phi=0$ (note that $\partial_{a} \Lambda^{\mu}=0$ is one of the equations of motion). Thus, Eq. (7) includes 12 essential parameters which correspond to the primary first class constraints $p_{0}^{\mu} \approx 0, \pi_{\phi} \approx 0$ in the Hamilton formalism (see below).

Let us consider the theory in the Hamiltonian framework. Momenta conjugate to the variables $\Lambda^{\mu}, A_{a}^{\mu}, \phi$ are denoted by $p_{\Lambda}^{\mu}, p_{a}^{\mu}, \pi_{\phi}$. All equations for determining the momenta turn out to be the primary constraints

$$
\begin{aligned}
& \pi_{\phi}=0, \\
& p_{0}^{\mu}=0 ; \\
& p_{\Lambda}^{\mu}=0, \\
& p_{1}^{\mu}-\Lambda^{\mu}=0 .
\end{aligned}
$$

The canonical Hamiltonian is

$$
H=\int d \sigma^{1}\left[\Lambda^{\mu} \partial_{1} A_{0}^{\mu}+\frac{1}{\phi} \Lambda^{2}+\lambda_{\phi} \pi_{\phi}+\lambda_{\Lambda}^{\mu} p_{\Lambda}^{\mu}+\lambda_{0}^{\mu} p_{0}^{\mu}+\lambda_{1}^{\mu}\left(p_{1}^{\mu}-\Lambda^{\mu}\right)\right],
$$

where $\lambda_{*}$ are the Lagrange multipliers corresponding to the constraints. The preservation in time of the primary constraints implies the secondary ones

$$
\begin{aligned}
& \partial_{1} \Lambda^{\mu}=0, \\
& \Lambda^{2}=0,
\end{aligned}
$$

and equations for determining some of the Lagrange multipliers

$$
\begin{aligned}
& \lambda_{1}^{\mu}=\partial_{1} A_{0}^{\mu}+\frac{2}{\phi} \Lambda^{\mu}, \\
& \lambda_{\Lambda}^{\mu}=0 .
\end{aligned}
$$

The tertiary constraints are absent.

Constraints (9) form a system of second class and can be omitted after introducing the corresponding Dirac bracket (the Dirac brackets for the remaining variables prove to coincide with the Poisson ones). After imposing the gauge fixing conditions $\phi=2, A_{0}^{\mu}=0$ for the first class constraints (8), dynamics of the remaining variables is governed by the equations

$$
\dot{A}_{1}^{\mu}=p_{1}^{\mu},
$$




$$
\begin{aligned}
& \dot{p}_{1}^{\mu}=0 \\
& \left(p_{1}^{\mu}\right)^{2}=0 \\
& \partial_{1} p_{1}^{\mu}=0 .
\end{aligned}
$$

In order to find a correct gauge for the second constraint in Eq.(14), let us consider Fourier decomposition of functions periodical in the interval $\sigma \subset[0, \pi]$

$$
\begin{aligned}
& A_{1}^{\mu}(\tau, \sigma)=Y^{\mu}(\tau)+\sum_{n \neq 0} y_{n}^{\mu}(\tau) e^{i 2 n \sigma} \\
& p_{1}^{\mu}(\tau, \sigma)=P_{y}^{\mu}(\tau)+\sum_{n \neq 0} p_{n}^{\mu}(\tau) e^{i 2 n \sigma}
\end{aligned}
$$

Then the constraint $\partial_{1} p_{1}^{\mu}=0$ is equivalent to $p_{n}^{\mu}=0, n \neq 0$, and an appropriate gauge is $y_{n}^{\mu}=0$, or, in the equivalent form $\partial_{1} A_{1}^{\mu}=0$. Thus, physical degrees of freedom of the model are the zero modes 2 of these variables, and the corresponding dynamics is

$$
\begin{aligned}
& A_{1}^{\mu}(\tau, \sigma)=Y^{\mu}+P_{y}^{\mu} \tau \\
& p_{1}^{\mu}(\tau, \sigma)=P_{y}^{\mu}=\mathrm{const} \\
& \left(P_{y}\right)^{2}=0
\end{aligned}
$$

Since there are no of oscillator variables, the action (6) can be considered as describing a point-like object, which propagates freely according to Eq.(16). The only quantum state is its ground state $\mid p_{y 0}>$ with the mass $m_{y}^{2}=p_{y 0}^{2}=0$. In the result, these degrees of freedom do not make contributions into the state spectrum of the superstring (see Sec.4), and manifest themselves in additional degeneracy of the continuous part of the energy spectrum only. The action of such a kind was successfully used before26 27 in a different context.

Note that in the previous discussion it was assumed that variables of the theory are periodical in the interval $\sigma \subset[0, \pi]$. For an open world sheet, the stationarity condition $\delta S_{\Gamma}=0$ for the Hamiltonian action

$$
S_{\Gamma}=\int d^{2} \sigma\left[p_{A} \dot{q}^{A}-H(q, p)\right]
$$

yields

$$
\int d \tau\left(\left.\Lambda^{\mu} \delta A_{0}^{\mu}\right|_{\sigma=0} ^{\sigma=\pi}\right)=0
$$

\footnotetext{
${ }^{2}$ We are grateful to N. Berkovits and J. Gates for bringing this fact to our attention
} 
Since the variations $\left.\delta A_{0}^{\mu}\right|_{\sigma=0, \pi}$ are arbitrary, this equation requires $\left.\Lambda^{\mu}\right|_{\sigma=0, \pi}=0$. By virtue of Eq. (9) it leads to the trivial solution $\left.p_{1}^{\mu}\right|_{\sigma=0, \pi}=0$. In contrast, for the closed world sheet one has $\left.\delta \Gamma^{A}\right|_{\sigma=0}=\left.\delta \Gamma^{A}\right|_{\sigma=\pi}$ for any variable $\Gamma^{A}$ and Eq. (17) is automatically satisfied. Hence, the model (6) has nontrivial solution being determined on the closed world sheet only.

\section{Eleven dimensional superstring action and its local symmetries}

The $D=11$ action functional to be examined is

$$
\begin{aligned}
& S=\int d^{2} \sigma\left\{\frac{-g^{a b}}{2 \sqrt{-g}} \Pi_{a}^{\mu} \Pi_{b}^{\mu}-i \varepsilon^{a b} \partial_{a} x^{\mu}\left(\bar{\theta} \Gamma^{\mu} \partial_{b} \theta\right)-\right. \\
&\left.-i \Lambda^{\mu} \bar{\psi} \Gamma^{\mu} \theta-\frac{1}{\phi} \Lambda^{\mu} \Lambda^{\mu}-\Lambda^{\mu} \varepsilon^{a b} \partial_{a} A_{b}^{\mu}\right\},
\end{aligned}
$$

where $\theta, \psi$ are 32-component Majorana spinors and $\Pi_{a}^{\mu} \equiv \partial_{a} x^{\mu}-i \bar{\theta} \Gamma^{\mu} \partial_{a} \theta$. Let us mention the origin of the terms presented in Eq. (18). The first two terms are exactly GS-type superstring action written in eleven dimensions. The meaning of the last two terms has been explained in the previous section. The third and the fourth terms will supply the appearance of the equations $\Lambda_{\mu} \Gamma_{\mu} \theta=0$ and $\Lambda^{2}=0$. Thus, the variables $\bar{\psi}^{\alpha}$ and $\phi$ are, in fact, the Lagrange multipliers for these constraints.

Note also that the Wess-Zumino term in the $D=10$ GS action provides the appear-

ance of the local $\kappa$-symmetry ${ }^{9}$. In our model it plays a different role, as will be discussed below.

Let us make a comment on the local symmetries structure of the action (18). Local bosonic symmetries are $d=2$ reparametrizations (with the standard transformation laws for all variables except for the variable $\phi$, which transforms as a density: $\left.\phi^{\prime}\left(\sigma^{\prime}\right)=\operatorname{det}\left(\partial \sigma^{\prime} / \partial \sigma\right) \phi(\sigma)\right)$, Weyl symmetry, and the transformations with parameters $\xi^{\mu}\left(\sigma^{a}\right)$ and $\omega_{a}\left(\sigma^{b}\right)$ described in the previous Section.

There is also a fermionic symmetry with parameters $\chi^{\alpha}\left(\sigma^{a}\right)$ :

$$
\begin{aligned}
& \delta \bar{\psi}=\bar{\chi} \Gamma^{\mu} \Lambda_{\mu}, \\
& \delta \phi=-\phi^{2}(\bar{\chi} \theta),
\end{aligned}
$$

from which only 16 are essential on-shell since $\Lambda^{2}=0$. As shown below, reducibility of this symmetry make no special problem for covariant quantization. 
Let us present arguments that the action constructed describes a free theory. Equations of motion for the theory (18) are

$$
\begin{aligned}
& \Pi_{a}^{\mu} \Pi_{b}^{\mu}-\frac{1}{2} g_{a b}\left(g^{c d} \Pi_{c}^{\mu} \Pi_{d}^{\mu}\right)=0 \\
& \partial_{a}\left(\frac{g^{a b}}{\sqrt{-g}} \Pi_{b}^{\mu}+i \varepsilon^{a b} \bar{\theta} \Gamma^{\mu} \partial_{b} \theta\right)=0 \\
& 4 i \Pi_{b}^{\mu}\left(\Gamma^{\mu} P^{-b a} \partial_{a} \theta\right)_{\alpha}+\varepsilon^{a b} \theta^{\beta} \partial_{a} \theta^{\gamma} \partial_{b} \theta^{\delta} \Gamma_{\alpha(\beta}^{\mu} C \Gamma_{\gamma \delta)}^{\mu}+i \Lambda^{\mu}\left(\Gamma^{\mu} \psi\right)_{\alpha}=0 ; \\
& \Lambda^{\mu} \Gamma^{\mu} \theta=0 \\
& \Lambda^{2}=0 \\
& \partial_{a} \Lambda^{\mu}=0 \\
& \varepsilon^{a b} \partial_{a} A_{b}^{\mu}+\frac{2}{\phi} \Lambda^{\mu}+i \bar{\psi} \Gamma^{\mu} \theta=0
\end{aligned}
$$

where

$$
P^{-b a}=\frac{1}{2}\left(\frac{g^{b a}}{\sqrt{-g}}-\varepsilon^{b a}\right) .
$$

Multiplying Eq. (20c) by $\Lambda_{\mu} \Gamma^{\mu}$ one gets

$$
\left(\Lambda^{\mu} \Pi_{b}^{\mu}\right) P^{-b a} \partial_{a} \theta=0 .
$$

In the coordinate system where $\Lambda^{-}=1$, supplemented by the conformal gauge, it can be rewritten as

$$
\left(\partial_{0}+\partial_{1}\right) \theta=0
$$

from which it follows that any solution $\theta(\sigma)$ of the system $(20)$ obeys this free equation.

Thus, Eqs. $(20 \mathrm{a}-\mathrm{c})$ for the $g^{a b}, x^{\mu}, \theta^{\alpha}$ variables in fact coincide with those of the GS string and are accompanied by $\Lambda_{\mu} \Gamma^{\mu} \theta=0$. The latter reduces to $\Gamma^{+} \theta=0$ in the coordinate system chosen. In the result, one expects free dynamics in this sector provided that the conformal gauge has been assumed. In the next section we will rigorously prove this fact by direct calculations in the Hamiltonian framework.

\section{Analysis of dynamics}

From the explicit form of the action functional (18) it follows that the variable $\Lambda^{\mu}$ can be excluded by making use of its equation of motion. The Hamiltonian analog of the 
situation is a pair of second class constraints $p_{\Lambda}{ }^{\mu}=0, p_{1}{ }^{\mu}-\Lambda^{\mu}=0$, which can be omitted after introducing the associated Dirac bracket (see Sec. 2). The Dirac brackets for the remaining variables prove to coincide with the Poisson ones and the Hamiltonian looks like

$$
\begin{aligned}
H= & \int d \sigma^{1}\left\{-\frac{N}{2}\left(\hat{p}^{2}+\Pi_{1 \mu} \Pi_{1}^{\mu}\right)-N_{1} \hat{p}_{\mu} \Pi_{1}^{\mu}+p_{1 \mu}\left(\partial_{1} A_{0}^{\mu}+i \bar{\psi} \Gamma^{\mu} \theta\right)+\right. \\
& \left.+\frac{1}{\phi}\left(p_{1}^{\mu}\right)^{2}+\lambda_{\phi} \pi_{\phi}+\lambda_{0 \mu} p_{0}^{\mu}+\lambda^{a b}\left(\pi_{g}\right)_{a b}+\lambda_{\psi}^{\alpha} p_{\psi \alpha}+L_{\alpha} \lambda_{\theta}{ }^{\alpha}\right\}
\end{aligned}
$$

where $p^{\mu}, p_{0}^{\mu}, p_{1}^{\mu}, p_{\psi \alpha},\left(\pi_{g}\right)_{a b}$ are momenta conjugate to the variables $x^{\mu}, A_{0}^{\mu}, A_{1}^{\mu}, \psi_{\alpha}, g_{a b}$, respectively; $\lambda_{*}$ are Lagrange multipliers corresponding to the primary constraints. In Eq. (23) we also denoted

$$
\begin{aligned}
& N=\frac{\sqrt{-g}}{g^{00}}, \\
& N_{1}=\frac{g^{01}}{g^{00}}, \\
& \hat{p}^{\mu}=p^{\mu}-i \bar{\theta} \Gamma^{\mu} \partial_{1} \theta, \\
& L_{\alpha} \equiv p_{\theta \alpha}-i\left(p^{\mu}+\Pi_{1}^{\mu}\right)\left(\bar{\theta} \Gamma^{\mu}\right)_{\alpha}=0 .
\end{aligned}
$$

It is interesting to note that the fermionic constraints $L_{\alpha}=0$ obey the algebra (2) and, being considered on their own (without taking into account the constraints $\bar{\theta} \Gamma^{\mu} p_{1 \mu}=0$ which will arise below), form a system which has no definite class (this corresponds to the lack of $\kappa$-symmetry in the GS action written in eleven dimensions).

The conservation in time of the primary constraints implies the secondary ones

$$
\begin{aligned}
& \partial_{1} p_{1}^{\mu}=0 \\
& \left(p_{1}^{\mu}\right)^{2}=0 \\
& \left(\bar{\theta} \Gamma^{\mu}\right)_{\alpha} p_{1}^{\mu}=0 \\
& \left(\hat{p}^{\mu} \pm \Pi_{1}^{\mu}\right)^{2}=0 \\
& \left(\bar{\lambda}_{\theta} \Gamma^{\mu}\right)_{\alpha}\left(\hat{p}^{\mu}+\Pi_{1}^{\mu}\right)+i \bar{\theta}^{\gamma} \partial_{1} \theta^{\delta} \lambda_{\theta}^{\beta} \Gamma_{\gamma(\delta}^{\mu} C \Gamma_{\beta \alpha)}^{\mu}+\frac{1}{2}\left(\bar{\psi} \Gamma^{\mu}\right)_{\alpha} \Lambda^{\mu}- \\
& -\left(\partial_{1} \bar{\theta} \Gamma^{\mu}\right)_{\alpha}\left(N+N_{1}\right)\left(\hat{p}^{\mu}+\Pi_{1}^{\mu}\right)-\frac{1}{2}\left(\bar{\theta} \Gamma^{\mu}\right)_{\alpha} \partial_{1}\left(N \hat{p}^{\mu}+N_{1} \Pi_{1}^{\mu}\right)=0
\end{aligned}
$$

At the next step, there arises only one nontrivial equation. From the condition $\left\{\bar{\theta} \Gamma^{\mu} p_{1}^{\mu}, H\right\}=0$ one gets

$$
\left(\bar{\lambda}_{\theta} \Gamma^{\mu}\right)_{\alpha} p_{1}^{\mu}=0 .
$$


Equations (25), (26) are equivalent to

$$
\begin{aligned}
& \bar{\lambda}_{\theta}=\left(N+N_{1}\right) \partial_{1} \bar{\theta}+\frac{\tilde{\xi}}{2} \bar{\theta}, \\
& \tilde{S}_{\alpha} \equiv\left(\bar{\psi} \Gamma^{\mu}\right)_{\alpha} p_{1}^{\mu}+\left(\bar{\theta} \Gamma^{\mu}\right)_{\alpha} \tilde{D}^{\mu}=0,
\end{aligned}
$$

where we denoted

$$
\begin{aligned}
& \tilde{D}^{\mu}=\tilde{\xi}\left(\hat{p}^{\mu}+\Pi_{1}^{\mu}\right)-\partial_{1}\left(N \hat{p}^{\mu}+N_{1} \Pi_{1}^{\mu}\right), \\
& \tilde{\xi}=\frac{\partial_{1}\left(N \hat{p}^{\mu}+N_{1} \Pi_{1}^{\mu}\right) p_{1}^{\mu}}{\left(\hat{p}^{\mu}+\Pi_{1}^{\mu}\right) p_{1}^{\mu}}
\end{aligned}
$$

Thus, we have Eq. (27) for determining the Lagrange multiplier $\lambda_{\theta}$ and the tertiary constraint $\tilde{S}_{\alpha}=0$. One can check that there are no more constraints in the problem.

Hamiltonian equations of motion for the variables $\left(g^{a b},\left(\pi_{g}\right)_{a b}\right),\left(\phi, \pi_{\phi}\right), \quad\left(A_{0}^{\mu}, p_{0}^{\mu}\right)$, $\left(\psi^{\alpha}, p_{\psi}^{\alpha}\right)$ look as follows: $\partial_{0} q=\lambda_{q}, \partial_{0} p_{q}=0$, while for other variables one has

$$
\begin{aligned}
& \partial_{0} A_{1}^{\mu}=\partial_{1} A_{0}^{\mu}+\frac{2}{\phi} p_{1}^{\mu}+i \bar{\psi} \Gamma^{\mu} \theta, \\
& \partial_{0} p_{1}^{\mu}=0, \\
& \partial_{0} x^{\mu}=-N \hat{p}^{\mu}-N_{1} \Pi_{1}^{\mu}-i \bar{\theta} \Gamma^{\mu} \lambda_{\theta}, \\
& \partial_{0} p^{\mu}=-\partial_{1}\left(N \Pi_{1}^{\mu}+N_{1} \hat{p}^{\mu}\right)+i \bar{\theta} \Gamma^{\mu} \lambda_{\theta}, \\
& \partial_{0} \theta^{\alpha}=-\lambda_{\theta}^{\alpha} .
\end{aligned}
$$

Note that equations $\partial_{0} p_{\theta \alpha}=\ldots$ have been omitted since they follow from the constraints $L_{\alpha}=0$ and other equations.

To go further, note that the constraints $\left(\pi_{g}\right)_{a b}=0$ form a nonvanishing Poisson bracket with the $\tilde{S}_{\alpha}$ from Eq. (29). A modification which splits them out of other constraints is

$$
\left(\tilde{\pi}_{g}\right)_{a b} \equiv\left(\pi_{g}\right)_{a b}+\frac{1}{2\left(\hat{p}+\Pi_{1}\right) p_{1}}\left(p_{\psi} \Gamma^{\mu} \Gamma^{\nu} \theta\right)\left(\hat{p}^{\mu}+\Pi_{1}^{\mu}\right) T^{\nu}{ }_{a b},
$$

with $T_{a b}^{\nu}$ being defined by the equality $\left\{\left(\pi_{g}\right)_{a b}, \tilde{S}_{\alpha}\right\}=T^{\mu}{ }_{a b}\left(\bar{\theta} \Gamma^{\mu}\right)_{\alpha}$. Hence, the constraints $\left(\tilde{\pi}_{g}\right)_{a b}=0$ are first class and one can adopt the gauge choice $g^{a b}=\eta^{a b}$. The full set of constraints can now be rewritten in a more simple form

$$
\begin{aligned}
& \pi_{\phi}=0, \\
& p_{0}^{\mu}=0 ; \\
& \left(p_{1}^{\mu}\right)^{2}=0,
\end{aligned}
$$




$$
\begin{aligned}
& \partial_{1} p_{1}^{\mu}=0, \\
& \left(\hat{p}^{\mu} \pm \Pi_{1}^{\mu}\right)^{2}=0, \\
& L_{\alpha}=0, \\
& \bar{\theta} \Gamma^{\mu} p_{1 \mu}=0, \\
& p_{\psi \alpha}=0, \\
& S_{\alpha} \equiv \bar{\psi} \Gamma^{\mu} p_{1 \mu}+\left(\bar{\theta} \Gamma^{\mu}\right)_{\alpha} D_{\mu}=0 .
\end{aligned}
$$

where

$$
\begin{aligned}
& D^{\mu} \equiv \xi\left(\hat{p}^{\mu}+\Pi_{1}^{\mu}\right)-\partial_{1} p^{\mu} \\
& \xi \equiv \frac{\partial_{1} \hat{p}^{\mu} p_{1 \mu}}{\left(\hat{p}^{\nu}+\Pi_{1}^{\nu}\right) p_{1 \nu}}
\end{aligned}
$$

Now, let us impose gauge fixing conditions to the first class constraints (30.a). The choice consistent with the equations of motion is

$$
\begin{aligned}
& \phi=2, \\
& A_{0}^{\mu}=-i \int_{0}^{\sigma} d \sigma^{\prime} \bar{\psi} \Gamma^{\mu} \theta
\end{aligned}
$$

After that, dynamics for the remaining variables looks like

$$
\begin{aligned}
& \partial_{0} \psi^{\alpha}=\lambda_{\psi}^{\alpha}, \\
& \partial_{0} p_{\psi \alpha}=0, \\
& p_{\psi \alpha}=0, \\
& S_{\alpha}=0 ; \\
& \partial_{0} A_{1}^{\mu}=p_{1}^{\mu}, \\
& \partial_{0} p_{1}^{\mu}=0, \\
& \left(p_{1}^{\mu}\right)^{2}=0, \\
& \partial_{1} p_{1}^{\mu}=0 ; \\
& \partial_{0} x^{\mu}=-p^{\mu}, \\
& \partial_{0} p^{\mu}=-\partial_{1} \partial_{1} x^{\mu}, \\
& \left(\hat{p}^{\mu} \pm \Pi_{1}^{\mu}\right)^{2}=0 ; \\
& \partial_{0} \theta=-\partial_{1} \theta-\frac{\xi}{2} \theta, \\
& L_{\alpha}=0, \\
& \left(\bar{\theta} \Gamma^{\mu}\right)_{\alpha} p_{1 \mu}=0 .
\end{aligned}
$$


The sector (33.a) includes $32+16$ independent constraints from which the first class ones can be picked out as follows:

$$
\left(p_{\psi} \Gamma^{\mu}\right)_{\alpha} p_{1 \mu}=0
$$

As was mentioned above, reducibility of the constraints does not spoil the covariant quantization program. Actually, let us impose the following covariant (and redundant) gauge fixing conditions for the constraints (34):

$$
S_{\alpha}^{1} \equiv \frac{1}{\left(\hat{p}+\Pi_{1}\right) p_{1}} \bar{\psi} \Gamma^{\mu}\left(\hat{p}_{\mu}+\Pi_{1 \mu}\right)=0 .
$$

Then the set of equations $S_{\alpha}=0, S^{1}{ }_{\alpha}=0$ is equivalent to

$$
S^{\prime} \equiv \bar{\psi}-\frac{1}{2\left(\hat{p}+\Pi_{1}\right) p_{1}} \bar{\theta} \Gamma^{\mu} D_{\mu} \Gamma^{\nu}\left(\hat{p}_{\nu}+\Pi_{1 \nu}\right),
$$

the latter forms a nondegenerate Poisson bracket together with the constraints $p_{\psi \alpha}=0$

$$
\left\{p_{\psi \alpha}, S_{\beta}^{\prime}\right\}=-C_{\alpha \beta}
$$

After passing to the Dirac bracket associated with the second class functions $p_{\psi_{\alpha}}, S_{\alpha}^{\prime}$, the variables $\psi, p_{\psi}$ can be dropped.

To proceed further, we impose the gauge $\partial_{1} A_{1}^{\mu}=0$ for the constraints in Eq. (33b), and pass to an appropriately chosen coordinate system. By making use of the Lorentz transformation one can consider a coordinate system where $P_{y}^{\mu}=(1,0, \ldots, 0,1)$ (note, that it is admissible procedure within the canonical quantization approach since the Lorentz transformation is a particular example a canonical one). To get dynamics in the final form, we pass to the light-cone coordinates $x^{\mu} \rightarrow\left(x^{+}, x^{-}, x^{i}\right), i=1,2, \ldots, 8,10$, $\theta^{\alpha} \rightarrow\left(\theta_{a}, \bar{\theta}_{\dot{a}}^{\prime}, \theta_{a}^{\prime}, \bar{\theta}_{\dot{a}}\right), a, \dot{a}=1, \ldots, 8$ and impose the gauge fixing conditions

$$
\begin{aligned}
& x^{+}=P^{+} \tau, \\
& p^{+}=-P^{+}=\mathrm{const},
\end{aligned}
$$

to the Virasoro first class constraints remaining in Eq.(33c). The equation $\bar{\theta} \Gamma^{\mu} p_{1 \mu}=0$ acquires now the form $\Gamma^{+} \theta=0$ and it is easy to show that $32+16$ constraints $L_{\alpha}=0$, $\Gamma^{+} \theta=0$ are second class. A solution is $\theta^{\alpha}=\left(\theta_{a}, 0,0, \bar{\theta}_{\dot{a}}\right)$ with $\theta_{a}$ and $\bar{\theta}_{\dot{a}}$ being $S O(8)$ spinors of opposite chirality. In the gauge chosen, the relation $\left(\hat{p}^{\mu}+\Pi_{1}^{\mu}\right) p_{1 \mu} \neq 0$ holds which correlates with the assumption made above in Eqs. (32), (35). For the remaining variables one gets free field equations 


$$
\begin{aligned}
& \partial_{0} x^{i}=-p^{i}, \\
& \partial_{0} p^{i}=-\partial_{1} \partial_{1} x^{i} ; \\
& \left(\partial_{0}+\partial_{1}\right) \theta_{a}=0, \\
& \left(\partial_{0}+\partial_{1}\right) \bar{\theta}_{\dot{a}}=0 .
\end{aligned}
$$

Moreover, $\theta_{a}$ and $\bar{\theta}_{\dot{a}}$ form two pairs of selfconjugate variables under the Dirac bracket associated with the constraints from Eq. (33d)

$$
\begin{aligned}
\left\{\theta_{a}, \theta_{b}\right\} & =\frac{i}{\sqrt{8} P^{+}} \delta_{a b}, \\
\left\{\bar{\theta}_{\dot{a}}, \bar{\theta}_{\dot{b}}\right\} & =\frac{i}{\sqrt{8} P^{+}} \delta_{\dot{a} \dot{b}} .
\end{aligned}
$$

Let us look shortly at the spectrum of the theory. The ground state of the full theory $\left|p_{y 0}, p_{0}, 0>=\right| p_{y 0}>\left|p_{0}>\right| 0>$ is a direct product of vacua, where $P_{y}^{2}\left|p_{y 0}>=0,\right| p_{0}>$ is a vacuum for zero modes of the variables $x^{\mu}, p^{\mu}$, while through $\mid 0>$ are denoted vacua for bosonic and fermionic oscillator modes. From Eq.(40) it follows that zero modes of the $\theta_{a}, \bar{\theta}_{\dot{a}}$ variables form the Clifford algebra which is also symmetry algebra of a ground state. A representation space is 256-dimensional which corresponds to the spectrum of the $D=11$ supergravity 29 . The excitation levels are then obtained by acting with oscillators on the ground state. One notes that zero modes $Y^{\mu}, P_{y}^{\mu}$ manifest themselves in additional degeneracy of the continuous energy spectrum only.

\section{A comment on the Wess-Zumino term in the $D=11$ superstring action}

For the $D=10$ GS superstring the Wess-Zumino term provides the local $\kappa$-symmetry 11, 12 , which leads to free dynamics for physical variables. Since there is no $\kappa$-symmetry in our construction, it is interesting to elucidate the meaning of this term in the $D=11$ action suggested. Let us consider the action (18) with the second term omitted. Canonical analysis for this model turns out to be very similar to that made above and we present results only.

Instead of Eqs. (24), (28), (29) one finds

$$
\begin{aligned}
& L_{\alpha} \equiv p_{\theta \alpha}-i\left(\bar{\theta} \Gamma^{\mu}\right)_{\alpha} p^{\mu}=0, \\
& \bar{\lambda}_{\theta}=\frac{N\left(\Pi_{1} p_{1}\right)+N_{1}\left(p p_{1}\right)}{\left(p p_{1}\right)} \partial_{1} \bar{\theta} \\
& \tilde{S} \equiv\left[\left(p p_{1}\right) \bar{\psi}-\partial_{1} \bar{\theta} \Gamma^{\rho}\left(N \Pi_{1}^{\rho}+N_{1} p^{\rho}\right) \Gamma^{\nu} p^{\nu}\right] \Gamma^{\mu} p_{1}^{\mu}=0 .
\end{aligned}
$$


In the coordinate system where $P_{y}^{\mu}=(1,0, \ldots, 0,1)$ the analog of the equations (33c), (33d) reads

$$
\begin{aligned}
& \partial_{0} x^{\mu}=-p^{\mu}-i \frac{\partial_{1} x^{+}}{p^{+}}\left(\bar{\theta} \Gamma^{\mu} \partial_{1} \theta\right), \\
& \partial_{0} p^{\mu}=-\partial_{1} \Pi_{1}^{\mu}, \\
& \left(p^{\mu} \pm \Pi_{1}^{\mu}\right)^{2}=0 \\
& \partial_{0} \theta=-\frac{\partial_{1} x^{+}}{p^{+}} \partial_{1} \theta, \\
& L_{\alpha}=0, \\
& \Gamma^{+} \theta=0,
\end{aligned}
$$

provided that the conformal gauge has been chosen.

To impose a gauge for the first class constraints $\left(p^{\mu} \pm \Pi_{1}^{\mu}\right)^{2}=0$, consider one-parameter set of equations

$$
\begin{aligned}
& x^{+}=P^{+}(\tau+c \sigma), \\
& p^{+}=-P^{+}=\mathrm{const}, \\
& c=\text { const } \neq \pm 1,
\end{aligned}
$$

which leads to the following dynamics for variables of the physical sector:

$$
\begin{aligned}
& \partial_{0} x^{i}=-p^{i}, \\
& \partial_{0} p^{i}=-\partial_{1} \partial_{1} x^{i}, \\
& \left(\partial_{0}-c \partial_{1}\right) \theta=0 .
\end{aligned}
$$

One can check that it is impossible to get rid of the number $c$ by making use of some other gauge choice for $g^{a b}$ and $A_{1}^{\mu}$ variables.

Thus, omitting the Wess-Zumino term in Eq. (18) one arrives at the theory which possesses all the properties of the model (18) with the only modification in the last of Eqs. (39): $\left(\partial_{0}-c \partial_{1}\right) \theta=0$ with $c$ a constant. Depending on the gauge chosen it can take any value except $c= \pm 1$. Hence, the dynamics is not manifestly $d=2$ Poincaré covariant, provided that $\theta$ be a $d=2$ scalar. It is the Wess-Zumino term which corrects this inconsistency.

\footnotetext{
${ }^{3}$ The value $c= \pm 1$ is not admissible since in that case the Poisson bracket of the constraints $\left(p^{\mu} \pm \Pi_{1}^{\mu}\right)^{2}=0$ and the gauges (43) vanishes.
} 


\section{Off-shell realization of the $D=11$ super-Poincaré algebra}

It is convenient first to recall some facts relating to the $D=10$ GS superstring. Off-shell realization of the super Poincaré algebra for that case includes the Poincaré transformations accompanied by the supersymmetries

$$
\begin{aligned}
\delta \theta^{\alpha} & =\epsilon^{\alpha}, \\
\delta x^{\mu} & =-i \bar{\theta} \Gamma^{\mu} \epsilon .
\end{aligned}
$$

Being considered on their own, in the gauge $\Gamma^{+} \theta=0$ these transformations are reduced to trivial shifts for variables of the physical sector

$$
\begin{aligned}
\delta \bar{\theta}_{\dot{a}} & =\bar{\epsilon}_{\dot{a}}, \\
\delta x^{i} & =0 .
\end{aligned}
$$

To get on-shell realization of the supersymmetry algebra, one needs to consider a combination of the $\epsilon$ - and $\kappa$-transformations $\delta_{\epsilon}+\delta_{\kappa(\epsilon)}$, which does not violate the gauge $\Gamma^{+} \theta=0$. These transformations are (see, for example, Ref. 28)

$$
\begin{aligned}
\delta \bar{\theta}_{\dot{a}} & =\bar{\epsilon}_{\dot{a}}+\frac{1}{P+} \partial_{-} x^{i} \bar{\gamma}_{\dot{a} a}^{i} \epsilon_{a}, \\
\delta x^{i} & =-i \sqrt{2}\left(\bar{\theta} \bar{\gamma}^{i} \epsilon\right) .
\end{aligned}
$$

We turn now to the $D=11$ case. Off-shell realization of the super Poincaré algebra for the action (18) includes the Poincaré transformations in the standard realization and the following supersymmetries with 32-component spinor parameter $\epsilon^{\alpha}$ :

$$
\begin{aligned}
& \delta \theta=\tilde{\Lambda} \epsilon \\
& \delta x^{\mu}=-i \bar{\theta} \Gamma^{\mu} \tilde{\Lambda} \epsilon \\
& \delta A^{\mu}{ }_{a}=-2 i \epsilon_{a b} \frac{g^{b c}}{\sqrt{-g}}\left(\bar{\theta} \tilde{\Pi}_{c} \Gamma^{\mu} \epsilon\right)-2 i \partial_{a} x^{\nu}\left(\bar{\theta} \Gamma^{\nu} \Gamma^{\mu} \epsilon\right)-2(\bar{\theta} \epsilon)\left(\bar{\theta} \Gamma^{\mu} \partial_{a} \theta\right) \\
& \delta \bar{\psi}=i \epsilon^{a b}\left[\bar{\epsilon} \Gamma^{\mu}\left(\partial_{a} \bar{\theta} \Gamma^{\mu} \partial_{b} \theta\right)-2 \partial_{a} \bar{\theta}\left(\partial_{b} \bar{\theta} \epsilon\right)\right] \\
& \delta \phi=-i \phi^{2}(\bar{\psi} \epsilon)
\end{aligned}
$$

where $\tilde{\Lambda} \equiv \Lambda_{\mu} \Gamma^{\mu}, \tilde{\Pi}_{c} \equiv \Pi_{c}{ }^{\mu} \Gamma^{\mu}$. The action is invariant up to total derivative terms. These transformations are the analog of Eq. (45) since in the physical sector they are reduced to $\delta \theta_{a}=\sqrt{2} \epsilon_{a}^{\prime}, \delta \bar{\theta}_{\dot{a}}=-\sqrt{2} \bar{\epsilon}_{\dot{a}}^{\prime}, \delta x^{i}=0$.

To find a global supersymmetry of the action (18) corresponding to Eq. (47) let us consider the following ansatz: 


$$
\begin{aligned}
& \delta \theta=\tilde{\Lambda} \tilde{\Pi}_{c} \epsilon^{c} \\
& \delta \phi=-i \phi^{2}\left(\bar{\psi} \tilde{\Pi}_{c} \epsilon^{c}\right), \\
& \delta x^{\mu}=4 i\left(\Lambda \Pi_{c}\right)\left(\bar{\theta} \Gamma^{\mu} \epsilon^{c}\right)+2 i\left(\bar{\theta} \tilde{\Pi}_{c} \epsilon^{c}\right) \Lambda^{\mu}
\end{aligned}
$$

where we denoted

$$
\begin{aligned}
& \epsilon_{\alpha}^{a} \equiv P^{-a b} \epsilon_{\alpha b}, \\
& P^{-a b}=\frac{1}{2}\left(\frac{g^{a b}}{\sqrt{-g}}-\varepsilon^{a b}\right), \\
& \left(\Lambda \Pi_{c}\right) \equiv \Lambda^{\mu} \Pi_{c}{ }^{\mu} .
\end{aligned}
$$

Variation of the GS part of the action (18) under these transformations looks like

$$
\begin{aligned}
& \delta S_{G S}=\varepsilon^{a b}\left[-8\left(\bar{\theta} \Gamma^{\mu} \epsilon^{c}\right)\left(\partial_{a} \bar{\theta} \Gamma^{\mu} \partial_{b} \theta\right)\left(\Lambda \Pi_{c}\right)-4\left(\bar{\theta} \tilde{\Pi}_{c} \epsilon^{c}\right)\left(\partial_{a} \bar{\theta} \tilde{\Lambda} \partial_{b} \theta\right)+\right. \\
& \left.+2\left(\partial_{a} \bar{\theta} \Gamma^{\mu} \tilde{\Lambda} \tilde{\Pi}_{c} \epsilon^{c}\right)\left(\bar{\theta} \Gamma^{\mu} \partial_{b} \theta\right)+\left(\bar{\theta} \Gamma^{\mu} \tilde{\Lambda} \tilde{\Pi}_{c} \epsilon^{c}\right)\left(\partial_{a} \bar{\theta} \Gamma^{\mu} \partial_{b} \theta\right)\right]- \\
& -2 i P^{-b a}\left[4\left(\bar{\theta} \tilde{\Pi}_{c} \epsilon^{c}\right)\left(\partial_{a} \Lambda \Pi_{b}\right)+2\left(\partial_{a} \bar{\theta} \tilde{\Lambda} \epsilon^{c}\right)\left(\Pi_{b} \Pi_{c}\right)-\left(\bar{\theta} \tilde{\Lambda} \partial_{a} \tilde{\Pi}_{b} \tilde{\Pi}_{c} \epsilon^{c}\right)\right]
\end{aligned}
$$

After integrating by parts, reordering the $\tilde{\Lambda}$ and $\tilde{\Pi}$ terms and making use of the identities

$$
\begin{aligned}
& P^{-a b} P^{-c d}=P^{-c b} P^{-a d} \\
& \left(\partial_{a} \bar{\theta} \Gamma^{\mu} \partial_{b} \theta\right)\left(\Lambda \Pi_{c}\right)=-\frac{1}{2} \partial_{a} \bar{\theta} \Gamma^{\mu}\left\{\tilde{\Lambda}, \tilde{\Pi}_{c}\right\} \partial_{b} \theta
\end{aligned}
$$

it proves to be possible to represent all the terms in Eq. (51) either as $K \tilde{\Lambda} \theta$ or $\partial_{a} \Lambda^{\mu} T^{\mu a}$ with $K$ and $T$ being certain coefficient. These terms can evidently be canceled by appropriate variations of the $\bar{\psi}$ and $A_{\mu}^{a}$ variables. The final form for these variations is

$$
\begin{aligned}
& \delta A_{a}^{\mu}=8\left(\bar{\theta} \Gamma^{\rho} \epsilon^{c}\right)\left(\bar{\theta} \Gamma^{\mu} \Pi_{c}^{\nu} \Gamma^{\nu \rho} \partial_{a} \theta\right)-5\left(\bar{\theta} \tilde{\Pi}_{c} \epsilon^{c}\right)\left(\bar{\theta} \Gamma^{\mu} \partial_{a} \theta\right)- \\
& \left.-3 \bar{\theta} \Gamma^{\mu} \Gamma^{\nu} \tilde{\Pi}_{c} \epsilon^{c}\right)\left(\bar{\theta} \Gamma^{\nu} \partial_{a} \theta\right)-4 i \varepsilon_{a d} P^{-b d}\left[\left(\bar{\theta} \Gamma^{\mu} \epsilon^{c}\right)\left(\Pi_{b} \Pi_{c}\right)-\right. \\
& \left.-2\left(\bar{\theta} \tilde{\Pi}_{c} \epsilon^{c}\right) \Pi_{b}^{\mu}\right] \\
& \delta \bar{\psi}=i \varepsilon^{a b}\left\{2\left(\partial_{a} \bar{\theta} \tilde{\Pi}_{c} \epsilon^{c}\right) \partial_{b} \bar{\theta}-8\left(\partial_{a} \bar{\theta} \tilde{\Pi}_{c} \partial_{b} \theta\right) \bar{\epsilon}^{c}-8 \partial_{a}\left[\left(\bar{\theta} \Gamma^{\mu} \epsilon^{c}\right) \partial_{b} \bar{\theta} \Gamma^{\mu \nu} \Pi_{\nu}^{c}\right]+\right. \\
& \left.+5\left(\bar{\theta} \partial_{a} \tilde{\Pi}_{c} \epsilon^{c}\right) \partial_{b} \theta+3\left(\bar{\theta} \Gamma^{\mu} \partial_{b} \theta\right) \bar{\epsilon}^{c} \partial_{a} \tilde{\Pi}_{c} \Gamma^{\mu}+\left(\partial_{a} \bar{\theta} \Gamma^{\mu} \partial_{b} \theta\right) \bar{\epsilon}^{c} \tilde{\Pi}_{c} \Gamma^{\mu}\right\}- \\
& -2 i P^{-b a}\left[\bar{\epsilon}^{c} \partial_{a} \tilde{\Pi}_{c} \tilde{\Pi}_{b}-2 \bar{\epsilon}^{c} \Pi_{b} \partial_{a} \Pi_{c}\right] .
\end{aligned}
$$

Note that the complicated transformation law for the $\psi$-variable might be predicted, since one of the Lagrangian equations of motion is

$$
(\tilde{\Lambda} \psi)_{\alpha}=-4 \tilde{\Pi}_{b} P^{-b a} \partial_{a} \theta_{\alpha}+i \varepsilon^{a b} \theta^{\beta} \partial_{a} \theta^{\gamma} \partial_{b} \theta^{\delta} \Gamma_{\alpha(\beta}^{\mu}\left(C \Gamma^{\mu}\right)_{\gamma \delta)} \cdot
$$


Thus, transformation of the $\tilde{\Lambda} \psi$ part of the $\psi$-variable is dictated by this equation and the transformation laws for the $x$ and $\theta$ variables.

Being reduced to the physical sector, Eq. (49) looks as follows:

$$
\begin{aligned}
\delta \theta_{a} & =-\sqrt{2}\left(P^{+} \epsilon_{a}-\partial_{-} x^{i} \gamma_{a \dot{a}}^{i} \bar{\epsilon}_{\dot{a}}^{\prime}+\partial_{-} x^{10} \epsilon_{a}^{\prime}\right), \\
\delta \bar{\theta}_{\dot{a}} & =-\sqrt{2}\left(P^{+} \bar{\epsilon}_{\dot{a}}+\partial_{-} x^{i} \bar{\gamma}_{\dot{a} a}^{i} \epsilon_{a}^{\prime}-\partial_{-} x^{10} \bar{\epsilon}_{\dot{a}}^{\prime}\right), \\
\delta x^{i} & =2 \sqrt{2} i P^{+}\left(\theta \gamma^{i} \bar{\epsilon}^{\prime}-\bar{\theta} \bar{\gamma}^{i} \epsilon^{\prime}\right)
\end{aligned}
$$

and seems to be the analog of Eq. (47).

To summarize, in this paper we have suggested a super Poincaré invariant action for the superstring which classically exists in any spacetime dimension. As compare with GS formulation for $N=1, D=10$ superstring action, the only difference is an additional infinite degeneracy in the continuous part of the energy spectrum, related with the zero modes $Y^{\mu}, P_{y}^{\mu}$. Since supersymmetry is realised in the physical subspace (55), one also gets the corresponding representation in the space of functions on that subspace. This allows one to expect a supersymmetric spectrum of quantum states. Analysis of this situation in terms of oscillator variables as well as the critical dimension will be presented in a separate publication.

The authors are grateful to N. Berkovits, I.L. Buchbinder, J. Gates and D.M. Gitman for useful discussions. This work was supported by Joint DFG-RFBR project No 96-0200180G, INTAS Grant-96-0308 (A.D.), INTAS-RFBR Grant No 95-829 (A.G.) and by FAPESP (A.D. and A.G.).

Note added: After this work has been completed, there appeared a paper by Bars and Deliduman 30 where a covariant action for superstring in a space with the nonstandard signature $(D-2,2)$ was suggested.

\section{Appendix A:}

In this Appendix we describe the minimal spinor representation of the Lorentz group $S O(1,10)$ which is know to have dimension $2^{[D / 2]}$. For this aim, it suffices to find eleven $32 \times 32 \Gamma^{\mu}$-matrices satisfying the equation $\Gamma^{\mu} \Gamma^{\nu}+\Gamma^{\nu} \Gamma^{\mu}=-2 \eta^{\mu \nu}, \mu, \nu=0,1, \ldots, 10$,

$\eta^{\mu \nu}=(+,-, \ldots,-)$. A convenient way is to use the well known $16 \times 16 \Gamma$-matrices of $S O(1,9)$ group which we denote as $\Gamma_{\alpha \beta}^{m}, \tilde{\Gamma}^{m \alpha \beta}, m=0,1, \ldots, 9$. Their explicit form is:

$$
\Gamma^{0}=\left(\begin{array}{cc}
\mathbf{1}_{8} & 0 \\
0 & \mathbf{1}_{8}
\end{array}\right),
$$




$$
\begin{aligned}
\tilde{\Gamma}^{0} & =\left(\begin{array}{cc}
-\mathbf{1}_{8} & 0 \\
0 & -\mathbf{1}_{8}
\end{array}\right), \\
\Gamma^{i} & =\left(\begin{array}{cc}
0 & \gamma^{i} a \dot{a} \\
\bar{\gamma}_{\dot{a} a}^{i} & 0
\end{array}\right), \\
\tilde{\Gamma}^{i} & =\left(\begin{array}{cc}
0 & \gamma^{i} a \dot{a} \\
\tilde{\gamma}_{\dot{a} a}^{i} & 0
\end{array}\right), \\
\Gamma^{9} & =\left(\begin{array}{cc}
\mathbf{1}_{8} & 0 \\
0 & -\mathbf{1}_{8}
\end{array}\right), \\
\tilde{\Gamma}^{9} & =\left(\begin{array}{cc}
\mathbf{1}_{8} & 0 \\
0 & -\mathbf{1}_{8}
\end{array}\right),
\end{aligned}
$$

where $\gamma_{a \dot{a}}^{i}, \bar{\gamma}_{\dot{a} a}^{i} \equiv\left(\gamma_{a \dot{a}}^{i}\right)^{\mathrm{T}}$ are real $S O(8) \gamma$-matrices

$$
\gamma^{i} \bar{\gamma}^{j}+\gamma^{j} \bar{\gamma}^{i}=2 \delta^{i j} \mathbf{1}_{8},
$$

where $i, a, \dot{a}=1, \ldots, 8$. As a consequence, the matrices $\Gamma^{m}, \tilde{\Gamma}^{m}$ are real, symmetric and obey the algebra

$$
\left\{\Gamma^{m}, \tilde{\Gamma}^{n}\right\}=-2 \eta^{m n} \mathbf{1},
$$

where $\eta^{m n}=(+,-, \ldots,-)$. Then a possible realization for the $D=11 \Gamma$-matrices is

$$
\Gamma^{\mu}=\left\{\left(\begin{array}{cc}
0 & \Gamma^{m} \\
\tilde{\Gamma}^{m} & 0
\end{array}\right),\left(\begin{array}{cc}
\mathbf{1}_{16} & 0 \\
0 & -\mathbf{1}_{16}
\end{array}\right)\right\},
$$

where $\mu=0,1, \ldots, 10$. The properties of $\Gamma^{m}, \tilde{\Gamma}^{m}$ induce the following relations for $\Gamma^{\mu}$ :

$$
\begin{aligned}
& \left(\Gamma^{0}\right)^{\mathrm{T}}=-\Gamma^{0}, \\
& \left(\Gamma^{i}\right)^{\mathrm{T}}=-\Gamma^{i}, \\
& \left(\Gamma^{\mu}\right)^{*}=\Gamma^{\mu} \\
& \left\{\Gamma^{\mu}, \Gamma^{\nu}\right\}=-2 \eta^{\mu \nu} \mathbf{1}_{32},
\end{aligned}
$$

where $\eta^{\mu \nu}=(+,-, \ldots,-)$. The charge conjugation matrix

$$
\begin{aligned}
& C \equiv \Gamma^{0} \\
& C^{-1}=-C, \\
& C^{2}=-\mathbf{1}
\end{aligned}
$$


can be used to construct the symmetric matrices: $\left(C \Gamma^{\mu}\right)^{\mathrm{T}}=C \Gamma^{\mu}$.

The next step is to introduce the antisymmetrized products

$$
\Gamma^{\mu \nu}=\frac{1}{2}\left(\Gamma^{\mu} \Gamma^{\nu}-\Gamma^{\nu} \Gamma^{\mu}\right),
$$

which have the following explicit form in terms of the corresponding $S O(1,9)$ and $S O(8)$ matrices:

$$
\begin{aligned}
& \Gamma^{0 i}=\left(\begin{array}{cc}
\Gamma^{0 i} & 0 \\
0 & \tilde{\Gamma}^{0 i}
\end{array}\right)=\left(\begin{array}{cc|cc}
0 & \gamma^{i} & 0 \\
\bar{\gamma}^{i} & 0 & & \\
\hline 0 & 0 & -\gamma^{i} \\
0 & -\bar{\gamma}^{i} & 0
\end{array}\right), \\
& \Gamma^{09}=\left(\begin{array}{cc}
\Gamma^{09} & 0 \\
0 & \tilde{\Gamma}^{09}
\end{array}\right)=\left(\begin{array}{cc|c}
1 & 0 & 0 \\
0 & -1 & \\
\hline 0 & -1 & 0 \\
0 & 1
\end{array}\right) \text {, } \\
& \Gamma^{i j}=\left(\begin{array}{cc}
\Gamma^{i j} & 0 \\
0 & \tilde{\Gamma}^{i j}
\end{array}\right)=\left(\begin{array}{cc|c}
\gamma^{i j} & 0 & 0 \\
0 & \bar{\gamma}^{i j} & 0 \\
\hline 0 & \gamma^{i j} & 0 \\
0 & \bar{\gamma}^{i j}
\end{array}\right),
\end{aligned}
$$

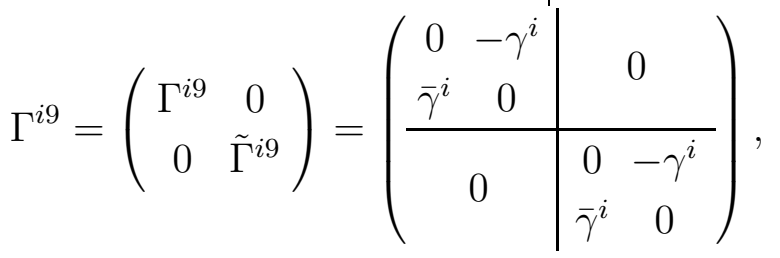

$$
\begin{aligned}
& \Gamma^{0,10}=\left(\begin{array}{cc}
0 & -\Gamma^{0} \\
\tilde{\Gamma}^{0} & 0
\end{array}\right)=\left(\begin{array}{c|cc}
0 & \mathbf{1} & 0 \\
& 0 & \mathbf{1} \\
\hline \mathbf{1} & 0 & 0 \\
0 & \mathbf{1} & 0
\end{array}\right), \\
& \Gamma^{i, 10}=\left(\begin{array}{cc}
0 & -\Gamma^{i} \\
\tilde{\Gamma}^{i} & 0
\end{array}\right)=\left(\begin{array}{c|cc}
0 & 0 & -\gamma^{i} \\
& -\bar{\gamma}^{i} & 0 \\
\hline 0 & \gamma^{i} & 0 \\
\bar{\gamma}^{i} & 0 & 0
\end{array}\right) \text {, } \\
& \Gamma^{9,10}=\left(\begin{array}{cc}
0 & -\Gamma^{9} \\
\tilde{\Gamma}^{9} & 0
\end{array}\right)=\left(\begin{array}{c|cc}
0 & -\mathbf{1} & 0 \\
& 0 & \mathbf{1} \\
\hline \mathbf{1} & 0 & 0 \\
0 & -\mathbf{1} & 0
\end{array}\right) \text {, }
\end{aligned}
$$


where $i=1,2, \ldots, 8$ and $\Gamma^{0 i}, \Gamma^{09}, \Gamma^{0,10}$ are symmetric, whereas $\Gamma^{i j}, \Gamma^{i 9}, \Gamma^{i, 10}, \Gamma^{9,10}$ are antisymmetric. Besides, these matrices are real and, as a consequence of Eq. (A5), obey the commutation relations of the Lorentz algebra.

Under the action of the Lorentz group a $D=11$ Dirac spinor is transformed as

$$
\delta \theta=\frac{1}{4} \omega_{\mu \nu} \Gamma^{\mu \nu} \theta
$$

Since the $\Gamma^{\mu \nu}$ matrices are real, the reality condition $\theta^{*}=\theta$ is compatible with Eq. (A10) which defines a Majorana spinor. To construct Lorentz-covariant bilinear combinations, note that

$$
\begin{aligned}
& \delta \bar{\theta}=-\frac{1}{4} \omega_{\mu \nu} \bar{\theta} \Gamma^{\mu \nu}, \\
& \bar{\theta} \equiv \theta^{\mathrm{T}} C .
\end{aligned}
$$

Then the combination $\bar{\psi} \Gamma^{\mu} \theta$ is a vector under the action of the $D=11$ Lorentz group

$$
\delta\left(\bar{\psi} \Gamma^{\mu} \theta\right)=-\omega^{\mu}{ }_{\nu}\left(\bar{\psi} \Gamma^{\mu} \theta\right)
$$

In various calculations the following properties:

$$
\begin{aligned}
& \bar{\psi} \Gamma^{\mu} \theta=-\bar{\theta} \Gamma^{\mu} \psi, \\
& \bar{\psi} \Gamma^{\mu} \Gamma^{\nu} \theta=\bar{\theta} \Gamma^{\nu} \Gamma^{\mu} \psi, \\
& \bar{\psi} \Gamma^{\mu} \Gamma^{\nu} \Gamma^{\rho} \theta=-\bar{\theta} \Gamma^{\rho} \Gamma^{\nu} \Gamma^{\mu} \psi
\end{aligned}
$$

are also useful.

It is possible to decompose a $D=11$ Majorana spinor in terms of its $S O(1,9)$ and $S O(8)$ components. Namely, from Eq. (A8) it follows that the decomposition

$$
\theta=\left(\bar{\theta}_{\alpha}, \theta^{\alpha}\right)
$$

where $\alpha=1, \ldots, 16$, holds. Here $\theta$ and $\bar{\theta}$ are Majorana-Weyl spinors of opposite chirality with respect to the $S O(1,9)$ subgroup of the $S O(1,10)$ group. Further, from the third equation in (A8) it follows that in the decomposition

$$
\theta=\left(\theta_{a}, \bar{\theta}_{\dot{a}}^{\prime}, \theta_{a}^{\prime}, \bar{\theta}_{\dot{a}}\right)
$$

where $a, \dot{a}=1, \ldots, 8$, the pairs $\theta_{a}, \theta_{a}^{\prime}$ and $\bar{\theta}_{\dot{a}}^{\prime}, \bar{\theta}_{\dot{a}}$ are $S O(8)$ spinors of opposite chirality. 
It is convenient to define the $D=11$ light-cone $\Gamma$-matrices

$$
\begin{aligned}
& \Gamma^{+}=\frac{1}{\sqrt{2}}\left(\Gamma^{0}+\Gamma^{9}\right)=\sqrt{2}\left(\begin{array}{cc|cc}
0 & \mathbf{1}_{8} & 0 \\
& 0 & 0 \\
\hline 0 & 0 & 0 \\
0 & -\mathbf{1}_{8} & 0
\end{array}\right), \\
& \Gamma^{-}=\frac{1}{\sqrt{2}}\left(\Gamma^{0}-\Gamma^{9}\right)=\sqrt{2}\left(\begin{array}{ccc}
0 & 0 & 0 \\
& 0 & \mathbf{1}_{8} \\
\hline-\mathbf{1}_{8} & 0 & 0 \\
0 & 0 & 0
\end{array}\right), \\
& \Gamma^{i}=\left(\begin{array}{cc}
0 & \Gamma^{i} \\
\tilde{\Gamma}^{i} & 0
\end{array}\right) \\
& \Gamma^{10}=\left(\begin{array}{cc}
\mathbf{1}_{16} & 0 \\
0 & -\mathbf{1}_{16}
\end{array}\right)
\end{aligned}
$$

where $i=1, \ldots, 8$. Then the equation $\Gamma^{+} \theta=0$ has a solution

$$
\theta=\left(\theta_{a}, 0,0, \bar{\theta}_{\bar{a}}\right) .
$$

Besides, under the condition $\Gamma^{+} \theta=0$ the following identities:

$$
\begin{aligned}
& \bar{\theta} \Gamma^{+} \partial_{1} \theta=\bar{\theta} \Gamma^{i} \partial_{1} \theta=\bar{\theta} \Gamma^{10} \partial_{1} \theta=0, \\
& \left(\bar{\theta} \Gamma^{\mu} \partial_{1} \theta\right) \Gamma^{\mu} \theta=0
\end{aligned}
$$

hold.

1. E. Witten, String Theory Dynamics in Various Dimensions, Nucl. Phys. B 443 (1995) 85.

2. M.J. Duff, $M$-Theory (The Theory Formerly Known as Strings), Int. J. Mod. Phys. A 11 (1996) 5623.

3. J.H. Schwarz, Lectures on Superstring and $M$-Theory Dualities, hep-th/9607201.

4. M.J. Duff, Supermembranes, hep-th/9611203.

5. P.K. Townsend, Four Lectures on M-Theory, hep-th/9612121.

6. E. Bergshoeff, E. Sezgin, and P.K. Townsend, Ann. of Physics, 185 (1988) 330. 
7. I. Bars, C.N. Pope, and E. Sezgin, Phys. Lett. B 198 (1987) 455.

8. M.J. Duff, P.S. Howe, T. Inami and K.S. Stelle, Phys. Lett. B 191 (1987) 70.

9. B. de Wit, J. Hoppe, and H. Nicolai, Nucl. Phys. B 305 (1988) 545.

10. B. de Wit, M. Luscher, and H. Nicolai, Nucl. Phys. B 320 (1989) 135.

11. M.B. Green and J.H. Schwarz, Phys. Lett. B 136 (1984) 367.

12. L. Brink and M. Henneaux, Principles of string theory, Plenum Press, New York and London, 1988.

13. T. Curtright, Phys. Rev. Lett. 60 (1987) 393.

14. E. Sezgin, Super $p$-Form Charges and Reformulation of the Supermembrane Action in Eleven Dimensions, hep-th/9512082.

15. A.A. Deriglazov and A.V. Galajinsky, A Linear Realization for the New Spacetime Superalgebras in Ten and Eleven Dimensions, Mod. Phys. Lett. A 12 (1997) 1517; hep-th/9703104.

16. N. Berkovits, A Problem with the Superstring Action of Deriglazov and Galajinsky, hepth/9712056.

17. H. Nishino and E. Sezgin, SYM Equations in 10+2 Dimensions, hep-th/9607185.

18. I. Bars, S-Theory, hep-th/9607112, hep-th/9607185.

19. I. Bars and C. Kounnas, A New Supersymmetry, hep-th/9612119; String and Particle with Two Times, hep-th/9705205.

20. I. Rudychev and E. Sezgin, Superparticles in $D>11$, hep-th/9704057.

21. P.K. Townsend, D-Branes from $M$-Branes, hep-th/9512062.

22. M. Cederwall, A. von Gussich, B.E.W. Nilsson, and A. Westenberg, The Dirichlet SuperThree-Brane in Ten-Dimensional Type IIB Supergravity, Nucl. Phys. B 490 (1997) 163.

23. M. Cederwall, A. von Gussich, B.E.W. Nilsson, P. Sindell, and A. Westenberg, The Dirichlet Super-p-Brane in Ten-Dimensional Type IIA and IIB Supergravity, Nucl. Phys. B 490 (1997) 179.

24. M. Aganagic, C. Popesku, and J.H. Schwarz, Gauge-Invariant and Gauge-Fixed D-brane Actions, Nucl. Phys. B 495 (1997) 99.

25. P. Pasti, D. Sorokin, and M. Tonin, Covariant Action for a $D=11$ Five-Brane with Chiral 
Field, Phys. Lett. B 398 (1997) 41.

26. A.A. Deriglazov and A.V. Galajinsky, Phys. Lett. B 386 (1996) 141.

27. A.A. Deriglazov and A.V. Galajinsky, Phys. Rev. D 54 (1996) 5195.

28. M. Kaku, Introduction to Superstrings, Springer-Verlag, 1988.

29. M.B. Green, J.H. Schwarz, and E. Witten, Superstring Theory, Cambridge Univ. Press, Cambridge, 1987.

30. I. Bars and C. Deliduman, Superstring with New Supersymmetry in $(9,2)$ and $(10,2)$ Dimensions, hep-th/9707215. 